\title{
Erratum to: An exploratory study of the worst-case scenario exercise as an exposure treatment for fear of cancer recurrence
}

\author{
Chelsea Moran $^{1,2} \cdot$ Christina Tomei $^{1} \cdot$ Monique Lefebvre $^{3}$. \\ Cheryl Harris $^{3}$ - Christine Maheu ${ }^{4} \cdot$ Sophie Lebel $^{1}$
}

Published online: 8 March 2017

(C) Springer-Verlag Berlin Heidelberg 2017

Erratum to: Support Care Cancer

DOI 10.1007/s00520-017-3600-4

Unfortunately, the original version of this article contained an error.

In Table 1 (pg. 2), line 2, it indicates "Time since diagnosis (years)" when it should say "Time since diagnosis (months)".

The online version of the original article can be found at http://dx. doi.org/10.1007/s00520-017-3600-4

\footnotetext{
Chelsea Moran

chelsea.moran2@mail.mcgill.ca

School of Psychology, University of Ottawa, Ottawa, ON, Canada

2 Department of Educational and Counselling Psychology, McGill University, Montreal, QC, Canada

3 Psychology Department, The Ottawa Hospital, Ottawa, ON, Canada

4 Ingram School of Nursing, McGill University, Montreal, QC, Canada
} 
Table 1 Demographic and medical characteristics of participants $(n=10)$

\begin{tabular}{lc}
\hline Characteristics & $M(\mathrm{SD})$ \\
\hline Age & $55.5(10.2)$ \\
Time since diagnosis (months) & $32.2(11.2)$ \\
& $\%$
\end{tabular}

Marital status

Single

Married/Common law $\quad 90$

Separated/Divorced 10

Widowed -

Ethnic background

Caucasian

Working status

Employed full time $\quad 50$

Employed part time by choice $\quad 10$

Employed part time due to illness -

Unemployed due to illness 10

Unemployed

Retired

Education

Some university/college $\quad 10$

University/college degree $\quad 60$

Graduate school $\quad 30$

Family income

$<\$ 20,000$

$\$ 21,000-40,000 \quad 10$

$\$ 41,000-60,000$

$\$ 61,000-80,000 \quad 30$

$\$ 81,000-100,000 \quad 10$

$>\$ 100,000 \quad 50$

Cancer diagnosis

$\begin{array}{ll}\text { Breast } & 70\end{array}$

Gynecological 30

Cancer stage (at diagnosis)

Stage I

Stage III 30

Treatments

$\begin{array}{ll}\text { Surgery } & 30\end{array}$

Chemotherapy and radiation $\quad 10$

Radiation and surgery $\quad 20$

Chemotherapy, radiation and surgery $\quad 40$ 